PROCEEDINGS OF THE

AMERICAN MATHEMATICAL SOCIETY

Volume 137, Number 7, July 2009, Pages 2451-2458

S 0002-9939(09)09876-1

Article electronically published on March 10, 2009

\title{
TWISTING QUASI-ALTERNATING LINKS
}

\author{
ABHIJIT CHAMPANERKAR AND ILYA KOFMAN \\ (Communicated by Daniel Ruberman)
}

\begin{abstract}
Quasi-alternating links are homologically thin for both Khovanov homology and knot Floer homology. We show that every quasi-alternating link gives rise to an infinite family of quasi-alternating links obtained by replacing a crossing with an alternating rational tangle. Consequently, we show that many pretzel links are quasi-alternating, and we determine the thickness of Khovanov homology for "most" pretzel links with arbitrarily many strands.
\end{abstract}

\section{INTRODUCTION}

In [15, quasi-alternating links were shown to be homologically thin for both Khovanov homology and knot Floer homology. It is the most inclusive known description of homologically thin links. However, the recursive definition makes it difficult to decide whether a given knot or link is quasi-alternating.

Definition 1.1 ([18]). The set $\mathcal{Q}$ of quasi-alternating links is the smallest set of links satisfying the following properties:

- The unknot is in $\mathcal{Q}$.

- If the link $\mathcal{L}$ has a diagram $L$ with a crossing $c$ such that

(1) both smoothings of $c, L_{0}$ and $L_{\infty}$ as in Figure 1 are in $\mathcal{Q}$, and

(2) $\operatorname{det}(L)=\operatorname{det}\left(L_{0}\right)+\operatorname{det}\left(L_{\infty}\right)$,

then $\mathcal{L}$ is in $\mathcal{Q}$.

We will say that $c$ as above is a quasi-alternating crossing of $L$ and that $L$ is quasi-alternating at $c$.

An oriented 3-manifold $Y$ is called an L-space if $b_{1}(Y)=0$ and $\left|H_{1}(Y ; \mathbb{Z})\right|=$ $r k \widehat{H F}(Y)$, where $\widehat{H F}$ denotes the Heegaard-Floer homology. For a link $\mathcal{L}$ in $S^{3}$, let $\Sigma(\mathcal{L})$ denote its branched double cover. The following property is the reason for the interest in quasi-alternating links:

Proposition 1.2 (Proposition 3.3 in [18]). If $\mathcal{L}$ is a quasi-alternating link, then $\Sigma(\mathcal{L})$ is an $\mathrm{L}$-space.

In this note, we show that a quasi-alternating crossing can be replaced by an alternating rational tangle to obtain another quasi-alternating link (Theorem 2.1 ). Thus, the set of quasi-alternating links includes many non-trivial infinite families.

Received by the editors April 22, 2008.

2000 Mathematics Subject Classification. Primary 57M25.

Key words and phrases. Khovanov homology, knot Floer homology, pretzel link.

The first author was supported by NSF grant DMS-0844485.

The second author was supported by NSF grant DMS-0456227 and a PSC-CUNY grant.

(c)2009 American Mathematical Society Reverts to public domain 28 years from publication 
For pretzel links, their Khovanov homology and knot Floer homology have been computed only for 3-strand pretzel links (see [17, 7, 21]). By repeatedly applying Theorem 2.1, we show that many pretzel links with arbitrarily many strands are quasi-alternating (Theorem 3.2(1)). Therefore, their respective homologies can be computed from the signature and the Jones and Alexander polynomials 11 Most other pretzel links are not quasi-alternating since their Khovanov homology lies on exactly three diagonals (Theorem $3.2(2)$ ). Thus, Theorem 3.2 gives the thickness of Khovanov homology for "most" pretzel links with arbitrarily many strands.

In Section 4, we apply Theorem 2.1 to complete the classification of quasialternating knots up to 10 crossings.

Finally, the Turaev genus plus two bounds the homological thickness for both Khovanov homology and knot Floer homology [16, 5, 13. The Turaev genus is preserved after inserting a rational tangle as in Theorem 2.1. We do not know of any quasi-alternating links with Turaev genus greater than one.

Question 1.3. Do there exist quasi-alternating links with arbitrary Turaev genus?

\section{TWISTING QUASI-ALTERNATING LINKS}

Let $c$ be a quasi-alternating crossing of a link diagram $L$, as in Definition [1.1 We consider $c$ as a 2-tangle with marked endpoints. Using Conway's notation for rational tangles, let $\varepsilon(c)= \pm 1$, according to whether the overstrand has positive or negative slope. We will say that a rational 2-tangle $\tau=C\left(a_{1}, \ldots, a_{m}\right)$ extends $c$ if $\tau$ contains $c$, and for all $i, \varepsilon(c) \cdot a_{i} \geq 1$. In particular, $\tau$ is an alternating rational tangle.

Theorem 2.1. If $L$ is a quasi-alternating link diagram, let $L^{\prime}$ be obtained by replacing any quasi-alternating crossing $c$ with an alternating rational tangle that extends c. Then $L^{\prime}$ is quasi-alternating.

We start with some background needed for the proof. For any connected link diagram $L$, we can associate a connected graph $G(L)$, called the Tait graph of $L$, by checkerboard coloring complementary regions of $L$, assigning a vertex to every shaded region, an edge to every crossing and a \pm sign to every edge as follows:
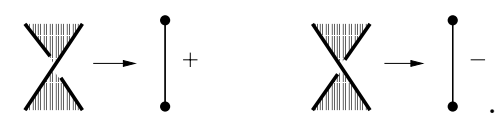

The signs are all equal if and only if $L$ is alternating.

Lemma 2.2. For any spanning tree $T$ of $G(L)$, let $v(T)$ be the number of positive edges in $T$. Let $s_{v}(L)=\#\{$ spanning trees $T$ of $G(L) \mid v(T)=v\}$. Then

$$
\operatorname{det}(L)=\left|\sum_{v}(-1)^{v} s_{v}(L)\right| .
$$

Proof. Thistlethwaite 22] gave an expansion of the Jones polynomial $V_{L}(t)$ in terms of spanning trees $T$ of $G(L)$. Using this expansion in 4 for any spanning tree $T$, we defined a grading $u(T)$ such that

$$
V_{L}(t)=(-1)^{w} t^{(3 w+k) / 4} \sum_{T \subset G}(-1)^{u(T)} t^{u(T)-v(T)},
$$

\footnotetext{
${ }^{1}$ A closed formula for the Kauffman bracket of arbitrary pretzel links is given in [3]; for the Alexander polynomial, see 9 and the references therein.
} 


\section{火 $x \asymp$ 兴來}

Figure 1 . Crossing $c$ of $L$, its smoothings $L_{0}$ and $L_{\infty}, L^{2}$ and $L^{-2}$.

where $w$ is the writhe of $L$ and $k$ is a constant that depends on $G$ (see the proof of Proposition 2 in [4]). Thus,

$$
\operatorname{det}(L)=\left|V_{L}(-1)\right|=\left|\sum_{T \subset G}(-1)^{u(T)}(-1)^{u(T)-v(T)}\right|=\left|\sum_{v \geq 0}(-1)^{v} s_{v}(L)\right|
$$

Theorem 2.1. Let $L$ be a quasi-alternating diagram at crossing $c$. We may assume that $\varepsilon(c)=1$ by rotating $L$ as needed. For $n \in \mathbb{Z}$, let $L^{n}$ denote the link diagram with $|n|$ additional crossings inserted at $c$, which are vertical positive half-twists if $n>0$ and horizontal negative half-twists if $n<0$. The cases $n= \pm 2$ are shown in Figure 1.

We first show that $L^{n}$ is quasi-alternating at any inserted crossing. Suppose $n \geq 0$. We checkerboard color $L$ such that the edge $e$ in $G(L)$ that corresponds to $c$ is positive. With the induced checkerboard coloring, the Tait graph $G\left(L_{0}\right)$ has the edge $e$ contracted, $G\left(L_{\infty}\right)$ has the edge $e$ deleted, and $G\left(L^{n}\right)$ has $n$ additional vertices on $e$, dividing it into $n+1$ positive edges $\left\{e_{0}, \ldots, e_{n}\right\}$.

For every spanning tree $T$ of $G(L)$ such that $e \in T$, there is a unique spanning tree $T^{\prime}$ of $G\left(L_{0}\right)$ and a unique spanning tree $T^{\prime \prime}$ of $G\left(L^{n}\right)$ such that $\left\{e_{0}, \ldots, e_{n}\right\} \subset T^{\prime \prime}$. Then $v\left(T^{\prime \prime}\right)=v(T)+n=v\left(T^{\prime}\right)+1+n$.

For every spanning tree $T$ of $G(L)$ such that $e \notin T$, there is a unique spanning tree $T^{\prime}$ of $G\left(L_{\infty}\right)$ and there are spanning trees $T_{0}, \ldots, T_{n}$ of $G\left(L^{n}\right)$ such that $e_{i} \notin T_{i}$. Then for $0 \leq i \leq n, v\left(T_{i}\right)=v(T)+n=v\left(T^{\prime}\right)+n$. For each $v$,

$$
s_{v}\left(L^{n}\right)=s_{v-n-1}\left(L_{0}\right)+(n+1) s_{v-n}\left(L_{\infty}\right) .
$$

Therefore,

$$
\sum_{v}(-1)^{v} s_{v}\left(L^{n}\right)=\sum_{v}(-1)^{v} s_{v-n-1}\left(L_{0}\right)+(n+1) \sum_{v}(-1)^{v} s_{v-n}\left(L_{\infty}\right) .
$$

By (2.1) with $n=0, s_{v}(L)=s_{v-1}\left(L_{0}\right)+s_{v}\left(L_{\infty}\right)$. Hence

$$
\sum_{v}(-1)^{v} s_{v}(L)=\sum_{v}(-1)^{v} s_{v-1}\left(L_{0}\right)+\sum_{v}(-1)^{v} s_{v}\left(L_{\infty}\right) .
$$

Let $x=\sum_{v}(-1)^{v} s_{v-1}\left(L_{0}\right)$ and $y=\sum_{v}(-1)^{v} s_{v}\left(L_{\infty}\right)$, so $\operatorname{det}\left(L_{0}\right)=|x|$ and $\operatorname{det}\left(L_{\infty}\right)=|y|$. Since $L$ is quasi-alternating at $c, \operatorname{det}(L)=\operatorname{det}\left(L_{0}\right)+\operatorname{det}\left(L_{\infty}\right)$; that is, $|x+y|=|x|+|y|$. Therefore, $x \cdot y \geq 0$. It now follows from (2.2) for $n \geq 0$ that

$$
\begin{aligned}
\left|\sum_{v}(-1)^{v} s_{v}\left(L^{n}\right)\right| & =\left|(-1)^{n} x+(n+1)(-1)^{n} y\right|=|x|+(n+1)|y|, \\
\operatorname{det}\left(L^{n}\right) & =\operatorname{det}\left(L_{0}\right)+(n+1) \operatorname{det}\left(L_{\infty}\right) .
\end{aligned}
$$




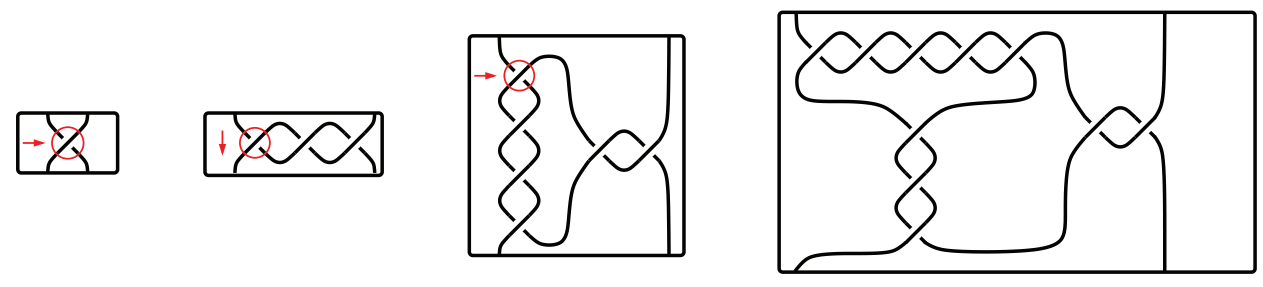

Figure 2. Crossing $c$ with $\varepsilon(c)=1$ is replaced by $C(5,3,2)$. The steps shown are $L \rightarrow L^{-2} \rightarrow\left(L^{-2}\right)^{3} \rightarrow\left(\left(L^{-2}\right)^{3}\right)^{-4}$.

Let $c$ denote any crossing in $L^{n}$ added to $L$ as above. Let $L_{0}^{n}$ and $L_{\infty}^{n}$ denote the corresponding resolutions of $L^{n}$ at $c$. We have $L_{\infty}^{n}=L_{\infty}$ and $L_{0}^{n}=L^{n-1}$ as links. For $n \geq 1$, (2.4) implies

$$
\begin{aligned}
\operatorname{det}\left(L^{n}\right) & =\operatorname{det}\left(L_{0}\right)+(n+1) \operatorname{det}\left(L_{\infty}\right) \\
& =\left(\operatorname{det}\left(L_{0}\right)+n \operatorname{det}\left(L_{\infty}\right)\right)+\operatorname{det}\left(L_{\infty}\right) \\
& =\operatorname{det}\left(L^{n-1}\right)+\operatorname{det}\left(L_{\infty}^{n}\right) \\
& =\operatorname{det}\left(L_{0}^{n}\right)+\operatorname{det}\left(L_{\infty}^{n}\right) .
\end{aligned}
$$

We have that $L^{0}=L$ and $L_{\infty}^{n}=L_{\infty}$ as links and hence they are quasialternating. If $L^{n-1}=L_{0}^{n}$ is quasi-alternating, then $L^{n}$ is quasi-alternating by the equations above. By induction, $L^{n}$ is quasi-alternating at $c$, so $L^{n}$ is quasialternating at every inserted crossing.

Suppose $n \leq 0$. If $L$ is quasi-alternating at $c$, then the mirror image $\tilde{L}$ is also quasi-alternating at $c$ since $\operatorname{det}(\tilde{L})=\operatorname{det}(L)$. Applying the argument above to $\tilde{L}$ and then reflecting prove this case for $L$.

Since every inserted crossing is quasi-alternating in $L^{n}$, we can iterate the construction above. Let $\tau=C\left(a_{1}, \ldots, a_{m}\right)$ be an alternating rational tangle that extends $c$. Since $\varepsilon(c) \cdot a_{i} \geq 1$ for all $i, L^{a_{i}}$ is a vertical positive twist operation, and $L^{-a_{i}}$ is a horizontal negative twist operation. We now construct:

$$
L^{\prime}=\left(\left(\left(L^{-a_{m}}\right)^{a_{m-1}}\right)^{-a_{m-2}} \cdots\right)^{(-1)^{m}\left(a_{1}-\varepsilon(c)\right)} .
$$

For example, see Figure 2, The resulting link $L^{\prime}$ is quasi-alternating and is obtained from $L$ by replacing $c$ with $C\left(a_{1}, a_{2}, \ldots, a_{m}\right)$.

The following lemma will be used in the next section.

Lemma 2.3. If $K$ and $L$ are any quasi-alternating knot diagrams, then $K \# L$ is quasi-alternating.

Proof. The proof is by induction on $\operatorname{det}(K)$. For quasi-alternating $K$, if $\operatorname{det}(K)=1$, then $K$ is the unknot, so the result follows. Otherwise, $K$ is quasi-alternating at a crossing $c$, so the two smoothings at $c, K_{0}$ and $K_{\infty}$, are quasi-alternating.

Since $\operatorname{det}(K)=\operatorname{det}\left(K_{0}\right)+\operatorname{det}\left(K_{\infty}\right), \operatorname{both} \operatorname{det}\left(K_{0}\right)<\operatorname{det}(K)$ and $\operatorname{det}\left(K_{\infty}\right)<$ $\operatorname{det}(K)$. By induction, both $K_{0} \# L$ and $K_{\infty} \# L$ are quasi-alternating. Moreover,

$$
\begin{aligned}
\operatorname{det}(K \# L) & =\operatorname{det}(K) \operatorname{det}(L)=\left(\operatorname{det}\left(K_{0}\right)+\operatorname{det}\left(K_{\infty}\right)\right) \operatorname{det}(L) \\
& =\operatorname{det}\left(K_{0}\right) \operatorname{det}(L)+\operatorname{det}\left(K_{\infty}\right) \operatorname{det}(L) \\
& =\operatorname{det}\left(K_{0} \# L\right)+\operatorname{det}\left(K_{\infty} \# L\right) .
\end{aligned}
$$

Therefore, $K \# L$ is quasi-alternating at $c$. 


\section{Pretzel Links}

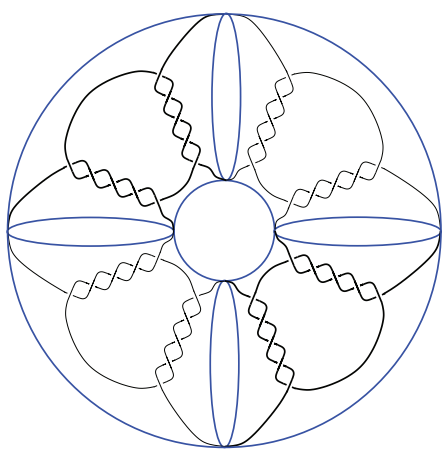

Alternating links are quasi-alternating, so we consider only non-alternating pretzel links. For all $p_{i}, q_{j} \geq 1$, let $P\left(p_{1}, \ldots, p_{n},-q_{1}, \ldots,-q_{m}\right)$ denote the $(m+n)$-strand pretzel link. As shown in the figure on the left, the standard diagram of a non-alternating pretzel link can be made alternating on the torus and so has Turaev genus one.

Proposition 3.1. If $m, n \geq 2$ and all $p_{i}, q_{j} \geq 2$, then the Khovanov homology of $P\left(p_{1}, \ldots, p_{n},-q_{1}, \ldots-q_{m}\right)$ has thickness exactly 3 . More generally, any nonalternating Montesinos link, obtained by replacing $p_{i}>1$ (resp. $q_{j}>1$ ) half-twists with a rational tangle that extends at least two of these crossings, also has thickness exactly 3.

Proof. It is easy to check that for $n, m \geq 2$ and $p_{i}, q_{j} \geq 2$, the standard diagram for $P\left(p_{1}, \ldots, p_{n},-q_{1}, \ldots,-q_{m}\right)$ is adequate. By Proposition 5.1 of [11, it has thick Khovanov homology. Since the Turaev genus of non-alternating pretzel links is one, the thickness bounds for Khovanov homology given in [5, 16] are achieved by pretzel links whose Khovanov homology is thick. Since adequacy (and Turaev genus) is preserved after replacing any half-twists with a rational tangle that extends at least two of these crossings, the same argument applies to these Montesinos links.

Similarly, it follows that every non-alternating Montesinos link (see 2] for their classification) has Turaev genus one. However, some of these have thin Khovanov homology.

Theorem 3.2. (1) For $n \geq 1, p_{i} \geq 1$ for all $i$, and $q>\min \left(p_{1}, \ldots, p_{n}\right)$, the pretzel link $P\left(p_{1}, \ldots, p_{n},-q\right)$ is quasi-alternating.

(2) For $m, n \geq 2$ and all $p_{i}, q_{j} \geq 2$, the pretzel link $P\left(p_{1}, \ldots, p_{n},-q_{1}, \ldots-q_{m}\right)$ is not quasi-alternating.

Both statements are true for all permutations of $p_{i}$ 's and $q_{j}$ 's and for reflections of all these pretzel links.

Proof. We first prove part (1) by induction on $n$. For $n=1, P\left(p_{1},-q\right)=$ $T\left(2, p_{1}-q\right)$, which is quasi-alternating since $p_{1}-q \neq 0$.

Let $n \geq 1$ and $p_{i} \geq 1$ for all $i$. Suppose for $q>\min \left(p_{1}, \ldots, p_{n}\right), P\left(p_{1}, \ldots, p_{n},-q\right)$ is quasi-alternating. Consider $P\left(p_{1}, \ldots, p_{n}, p_{n+1},-q\right)$. Without loss of generality, $q>p_{i_{0}}$ for some $i_{0}$, with $1 \leq i_{0} \leq n$. Let $L=P\left(p_{1}, \ldots, p_{n}, 1,-q\right)$, and let $c$ be the crossing on the $(n+1)$ st strand. Let $T(2, k)$ denote the alternating torus link. Then $L_{0}=T\left(2, p_{1}\right) \# \ldots \# T\left(2, p_{n}\right) \# T(2,-q)$ is quasi-alternating by Lemma 2.3 . and $L_{\infty}=P\left(p_{1}, \ldots, p_{n},-q\right)$ is quasi-alternating by the induction hypothesis.

By Lemma 4.3 of [6],

$$
\operatorname{det}\left(P\left(p_{1}, \ldots, p_{n},-q_{1}, \ldots,-q_{m}\right)\right)=\left|\prod_{i=1}^{n} p_{i} \prod_{j=1}^{m} q_{j}\left(\sum_{i=1}^{n} \frac{1}{p_{i}}-\sum_{j=1}^{m} \frac{1}{q_{j}}\right)\right| .
$$


Now, $q>p_{i_{0}}$ implies $\frac{1}{p_{i_{0}}}-\frac{1}{q}>0$, so

$$
q \prod_{i=1}^{n} p_{i}\left(1+\sum_{i \neq i_{0}} \frac{1}{p_{i}}+\left(\frac{1}{p_{i_{0}}}-\frac{1}{q}\right)\right)=q \prod_{i=1}^{n} p_{i}\left(\sum_{i \neq i_{0}} \frac{1}{p_{i}}+\left(\frac{1}{p_{i_{0}}}-\frac{1}{q}\right)\right)+q \prod_{i=1}^{n} p_{i} .
$$

Therefore, $\operatorname{det}(L)=\operatorname{det}\left(L_{0}\right)+\operatorname{det}\left(L_{\infty}\right)$, which proves that $L=P\left(p_{1}, \ldots, p_{n}, 1,-q\right)$ is quasi-alternating at $c$. By Theorem 2.1, $L=P\left(p_{1}, \ldots, p_{n}, p_{n+1},-q\right)$ is quasialternating for all $p_{n+1} \geq 1$. This completes the proof of part (1) by induction.

By Proposition 3.1 the pretzel links in part (2) have thick Khovanov homology, so they are not quasi-alternating.

The arguments above remain essentially unchanged for all permutations of $p_{i}$ 's and $q_{j}$ 's and for all reflections, given by negating every $p_{i}$ and $q_{j}$.

Remark 3.3. Widmer 23 extended Theorem 3.2 to certain Montesinos links.

3.1. 3-strand pretzel links. Theorem 3.2 still leaves open the quasi-alternating status of many pretzel links. For certain 3-strand pretzel links, this can be deduced from previous results. As above, the statements below for $P\left(p_{1}, p_{2},-q\right)$ are true for all permutations of $p_{i}$ 's and $q$ and for reflections of all these pretzel links.

According to [8, 12], for the pretzel link $L=P\left(p_{1}, p_{2},-q\right)$ with $p_{1}, p_{2}, q \geq 2$, $\Sigma(L)$ is an L-space if and only if

(1) $q \geq \min \left\{p_{1}, p_{2}\right\}$ or

(2) $q=\min \left\{p_{1}, p_{2}\right\}-1$ and $\max \left\{p_{1}, p_{2}\right\} \leq 2 q+1$.

By Proposition 1.2, 3-strand pretzel links that satisfy neither (1) nor (2) above are not quasi-alternating. Together with Theorem $3.2(1)$, this leaves open the quasialternating status for the following 3 -strand pretzel links:

Question 3.4. (1) For $p \geq q \geq 2$, is $P(p, q,-q)$ quasi-alternating?

(2) For $2 q+1 \geq p \geq q+1 \geq 3$, is $P(p, q+1,-q)$ quasi-alternating?

Note that $P(3,3,-3)=9_{46}$ and $P(4,3,-3)=10_{140}$. In addition, thick Khovanov homology or knot Floer homology precludes some of these links from being quasi-alternating (see [17, 7, 21]). For example, $P(k, 2,-2)$ has thick Khovanov homology for $2 \leq k \leq 5$ according to KhoHo [19].

\section{Quasi-ALternating KNOtS UP TO 10 CROSSINGS}

Manolescu [14] showed that all KH-thin knots up to 9 crossings, except $9_{46}$, are quasi-alternating. Among 42 non-alternating 10-crossing knots, 32 are KH-thin. Baldwin [1] showed that among these, 10 knots, which are closed 3-braids, are quasi-alternating. Greene [8] showed that 8 more knots- $10_{150}, 10_{151}, 10_{156}, 10_{158}$, $10_{163}, 10_{164}, 10_{165}, 10_{166}$ - are quasi-alternating. We show that except for $10_{140}$, the remaining 13 knots are quasi-alternating. In Table 1, we give the knot and its Conway notation (see [10]). For our computations, we replaced the rational tangle indicated in bold with a crossing of the same sign and checked that this crossing is quasi-alternating in the new diagram. It follows from Theorem 2.1 that the knot is quasi-alternating.

As a result, all $\mathrm{KH}$-thin knots up to 10 crossings, except $9_{46}$ and $10_{140}$, are quasi-alternating. Shumakovitch [20] informed us that $9_{46}$ and $10_{140}$ have thick odd Khovanov homology, so they are not quasi-alternating. 
TABLE 1

\begin{tabular}{|c|c||c|c||c|c|}
\hline $10_{129}$ & $\mathbf{3 2}, 21,2-$ & $10_{135}$ & $221, \mathbf{2 1}, 2-$ & $10_{146}$ & $22, \mathbf{2 1}, 21-$ \\
\hline $10_{130}$ & $311, \mathbf{3}, 2-$ & $10_{137}$ & $\mathbf{2 2}, 211,2-$ & $10_{147}$ & $211, \mathbf{3}, 21-$ \\
\hline $10_{131}$ & $311, \mathbf{2 1}, 2-$ & $10_{138}$ & $\mathbf{2 1 1}, 211,2-$ & $10_{160}$ & $-30: \mathbf{2 0}: 20$ \\
\hline $10_{133}$ & $\mathbf{2 3 , 2 1 , 2 -}$ & $10_{142}$ & $31, \mathbf{3}, 3-$ & & \\
\hline $10_{134}$ & $221, \mathbf{3}, 2-$ & $10_{144}$ & $31, \mathbf{2 1}, 21-$ & & \\
\hline
\end{tabular}

\section{ACKNOWLEDGEMENTS}

We thank Peter Ozsváth for useful discussions, which included the proof of Lemma 2.3. We thank Josh Greene for very helpful discussions about results in Sections 3.1 and 4. We thank Alex Shumakovitch for informing us about $9_{46}$ and $10_{140}$. We thank the anonymous referee for thoughtful comments.

\section{REFERENCES}

1. J. Baldwin, Heegaard Floer homology and genus one, one boundary component open books (arXiv:0804.3624v2 [math.GT]).

2. G. Burde and H. Zieschang, Knots, second ed., de Gruyter Studies in Mathematics, vol. 5, Walter de Gruyter \& Co., Berlin, 2003. MR1959408 (2003m:57005)

3. A. Champanerkar and I. Kofman, On links with cyclotomic Jones polynomials, Algebr. Geom. Topol. 6 (2006), 1655-1668. MR2253460 (2007f:57009)

4. __ Spanning trees and Khovanov homology, Proc. Amer. Math. Soc. 137 (2009), no. 6, $2157-2167$.

5. A. Champanerkar, I. Kofman, and N. Stoltzfus, Graphs on surfaces and Khovanov homology, Algebr. Geom. Topol. 7 (2007), 1531-1540. MR2366169 (2008m:57005)

6. O. T. Dasbach, D. Futer, E. Kalfagianni, X. S. Lin, and N. W. Stoltzfus, Alternating sum formulae for the determinant and other link invariants (arXiv:math/0611025v2 [math.GT]).

7. E. Eftekhary, Heegaard Floer homologies of pretzel knots (arXiv:math.GT/0311419).

8. J. Greene, A spanning tree model for the Heegaard Floer homology of a branched double-cover (arXiv:0805.1381v1 [math.GT]).

9. E. Hironaka, The Lehmer polynomial and pretzel links, Canad. Math. Bull. 44 (2001), no. 4, 440-451. MR1863636 (2002g:57009)

10. A. Kawauchi, A survey of knot theory, Birkhäuser Verlag, Basel, 1996; translated and revised from the 1990 Japanese original by the author. MR1417494 (97k:57011)

11. M. Khovanov, Patterns in knot cohomology. I, Experiment. Math. 12 (2003), no. 3, 365-374. MR2034399 (2004m:57022)

12. P. Lisca and A. Stipsicz, Ozsváth-Szabó invariants and tight contact three-manifolds. III, J. Symplectic Geom. 5 (2007), no. 4, 357-384. MR2413308

13. A. Lowrance, On knot Floer width and Turaev genus (arXiv:0709.0720v1).

14. C. Manolescu, An unoriented skein exact triangle for knot Floer homology, Math. Res. Lett. 14 (2007), no. 5, 839-852. MR.2350128 (2008m:57074)

15. C. Manolescu and P. Ozsváth, On the Khovanov and knot Floer homologies of quasialternating links (arXiv:0708.3249v2 [math.GT]).

16. V. Manturov, Minimal diagrams of classical and virtual links (arXiv:math.GT/0501393).

17. P. Ozsváth and Z. Szabó, Knot Floer homology, genus bounds, and mutation, Topology Appl. 141 (2004), no. 1-3, 59-85. MR2058681(2005b:57028)

18. no. 1, 1-33. MR2141852 (2006e:57041)

19. A. Shumakovitch, KhoHo, available from http://www.geometrie.ch/KhoHo/ (2003).

20. A. Shumakovitch, Private communication, March 2008.

21. R. Suzuki, Khovanov homology and Rasmussen's s-invariants for pretzel knots (arXiv:math.QA/0610913). 
22. M. Thistlethwaite, A spanning tree expansion of the Jones polynomial, Topology 26 (1987), 297-309. MR899051 (88h:57007)

23. T. Widmer, Quasi-alternating Montesinos links (arXiv:math/0811.0270 [math.GT]).

Department of Mathematics, College of Staten Island, The City University of New York, Staten Island, New York 10314

E-mail address: abhijit@math.csi.cuny.edu

Department of Mathematics, College of Staten Island, The City University of New York, Staten Island, New York 10314

E-mail address: ikofman@math.csi.cuny.edu 